\title{
INVENTÁRIO DE ESTILOS PARENTAIS (IEP) - Gomide (2006) PERCURSO DE PADRONIZAÇÃO E NORMATIZAÇÃO
}

\author{
Parenting Style Inventory (PSI) - Gomide (2006) \\ Normatization and Standardization Process
}

\author{
Izabela Tissot Antunes Sampaio ${ }^{1}$ \\ Paula Inez Cunha Gomide ${ }^{2}$
}

\section{Resumo}

Após oito anos de estudos acerca do tema, o Inventário de Estilos Parentais (IEP - Gomide, 2006) foi validado e encontra-se disponível para utilização. Esse instrumento avalia o estilo parental, ou seja, as estratégias e técnicas utilizadas pelos pais para educar os filhos, por meio de sete práticas educativas, sendo cinco vinculadas ao desenvolvimento do comportamento anti-social: negligência, punição inconsistente, disciplina relaxada, monitoria negativa e abuso físico; e duas relacionadas ao desenvolvimento de comportamentos prósociais: comportamento moral e monitoria positiva. Este artigo contempla os estudos-piloto, estudos sobre fidedignidade, validade interna, externa, perfil das mães de adolescentes infratores, estudos sobre comportamento moral, relação entre práticas educativas e abuso de drogas e a normatização dos dados obtidos. Da aplicação do IEP obtêm-se escores de cada uma das práticas e também o índice de estilo parental geral (IEP). O escore permite que o aplicador apreenda informações sobre as práticas educativas familiares utilizadas, facilitando a escolha dos procedimentos de intervenção (orientação, treinamento ou terapia) a serem utilizados com a família. O IEP é o primeiro instrumento psicológico brasileiro capaz de avaliar as práticas educativas parentais de crianças e adolescentes que apresentam risco.

Palavras-chave: Família; Práticas Educativas; Inventário de Estilos Parentais.

Mestranda do Programa de Pós-graduação em Psicologia da UFSC.

2 Doutora em Psicologia Experimental pela USP. Professora de Psicologia da Faculdade Evangélica do Paraná Endereço para correspondência: Rua do Viamão, 379, Guabirotuba, Curitiba- PR, CEP: 81520-380.

E-mail: izabelatissot@gmail.com 


\section{Abstract}

After eight years of studies on area, the Parenting Style Inventory (PSI - Gomide, 2006) has been validated and is now available for use. This instrument assesses the parental style, that is, strategies and techniques used by parents to raise their children, through seven educative practices, five of them related to antisocial behavior development: negligence, inconsistent punishment, lax discipline, negative monitoring and physical abuse; and two of them related to pro-social behaviors: moral modeling and positive monitoring. This work includes the pilot-studies, the studies about reliability, internal and external validity, law-breakers mothers' characteristics, moral modeling, the relation between educative practices and drug abuse, and data standardization. From the PSI application, scores of each educative practice are obtained and also the parental style index (psi). The score allows the applicator have information about the family educative practices, which facilitates intervention procedures (orientation, training or therapy) with the family. The PSI is the first Brazilian psychological instrument able to evaluate parental educative practices of children and adolescents at risk.

Keywords: Family; Educative Practices; Parenting Style Inventory.

\section{Introdução}

Segundo Cozby (2003), para se fazer pesquisa, por vezes temos que construir instrumentos que nos possibilitem a orientação de nossas idéias de maneira mais apurada, de forma a evitar ao máximo que nossas próprias crenças interfiram na coleta e análise dos dados. Assim, no campo do saber científico, nesse caso especial dentro da Psicologia, ao falarmos da padronização e normatização de uma medida psicológica, não podemos deixar de citar o percurso metodológico ao qual a construção de tal medida se submeteu.

Pasquali (2003) alerta que, comumente, os conceitos de padronização e normatização são utilizados como sinônimos na literatura, referindo-se à "necessidade de existir uniformidade em todos os procedimentos no uso de um teste válido e preciso; desde as precauções a serem tomadas na aplicação do teste (...) até o desenvolvimento de parâmetros ou critérios para a interpretação dos resultados obtidos" (p. 226).

No entanto, Pasquali (2003) distingue os conceitos de Padronização e Normatização. Para ele, no sentido mais técnico de parâmetro psicométrico, a padronização refere-se a como interpretar os resultados obtidos num teste. A padronização das condições de testagem, esclarece o autor, pretende garantir o uso adequado e legítimo dos testes psicológicos. A normatização, por sua vez, diz respeito aos padrões de como se deve interpretar um escore que o sujeito recebeu no teste, visto que um escore bruto obtido necessita ser contextualizado para poder ser interpretado.
O presente estudo objetiva apresentar $\mathrm{O}$ percurso percorrido para a Elaboração, Padronização e Normatização do Inventário de Estilos Parentais (Gomide, 2006), contemplando a justificativa social e científica para a elaboração do instrumento, os estudos-piloto para sua padronização, estudos sobre fidedignidade, validade interna e externa.

\section{Justificativa Social e Científica}

Aqueles que trabalham com famílias de risco social não encontram entre os instrumentos de avaliação disponíveis um que seja apropriado para medir os estilos parentais e orientar os profissionais para a correta atuação, seja na orientação, treinamento ou terapia para famílias que procuram o sistema jurídico e os programas de atenção à família apoiados pelas políticas públicas.

Da mesma forma, a literatura que trata dos Estilos Parentais voltados para o desenvolvimento de comportamentos pró-sociais e anti-sociais, embora vasta, não oferece um instrumento que avalie qual prática educativa precisa ser melhor orientada, ou que mostre aquelas que estão correlacionadas com o uso de drogas, comportamento infrator, parricídio e outros comportamentos anti-sociais, por exemplo (Gomide, 2003; Pettit, Laird, Dodge, Bates \& Criss, 2001; Reppold, Pacheco, Bardagi \& Hutz, 2002). 


\section{Modelo Teórico}

Gomide (2006) selecionou em seu modelo teórico sete práticas educativas que compõem o Estilo Parental, definido pela autora como o conjunto de práticas educativas utilizadas pelos pais na interação com os filhos; cinco delas estão relacionadas ao desenvolvimento de comportamentos anti-sociais (abuso físico, punição inconsistente, disciplina relaxada, monitoria negativa e negligência) e duas favoráveis ao desenvolvimento de comportamentos pró-sociais (monitoria positiva e comportamento moral).

A monitoria positiva é definida como o conjunto de práticas parentais que envolvem atenção e conhecimento dos pais acerca de onde seu filho se encontra e das atividades desenvolvidas por ele (Dishion \& McMahon, 1998; Stattin \& Kerr, 2000; Gomide, 2001, 2003). Para Gomide (2003), são ainda componentes da monitoria positiva as demonstrações de afeto e carinho dos pais, principalmente relacionados aos momentos de maior necessidade da criança.

O comportamento moral refere-se a uma prática educativa pela qual os pais transmitem valores, como honestidade, generosidade e senso de justiça aos filhos, auxiliando-os na discriminação do certo e do errado por meio de modelos positivos, dentro de uma relação de afeto. Pesquisas apontam alguns fatores como sendo essenciais para o desenvolvimento do comportamento moral nas crianças, são eles: a existência do sentimento de culpa; o desenvolvimento da empatia; ações honestas; crenças parentais positivas sobre trabalho e ausência de práticas anti-sociais (Araújo, 1999; Comte-Sponville, 2000; Hoffman, 1975; Gomide, 2003; Loos, Ferreira \& Vasconcelos, 1999; Patterson, Reid \& Dishion, 1992; Mussen, Conger \& Cagan, 1974).

A negligência ocorre quando os pais não estão atentos às necessidades de seus filhos, ausentam-se das responsabilidades, omitem-se de auxiliar seus filhos, ou simplesmente quando interagem sem afeto, sem amor. Segundo Dodge, Pettit e Battes (1994), a falta de calor e carinho na interação com a criança pode desencadear sentimentos de insegurança, vulnerabilidade e eventual hostilidade e agressão em relacionamentos sociais.

A punição inconsistente se dá quando os pais punem ou reforçam os comportamentos de seus filhos de acordo com o seu (dos pais) bom ou mau humor, de forma não contingente ao comportamento da criança. Assim, é o estado emocional dos pais que determina as ações educativas, e não as ações da criança. Como conseqüência, a criança aprende a discriminar o humor de seus pais e não aprende se seu ato foi adequado ou inadequado (Gomide, 2003).

A monitoria negativa (ou supervisão estressante) caracteriza-se pelo excesso de fiscalização dos pais sobre a vida dos filhos e pela grande quantidade de instruções repetitivas, as quais não são seguidas pelos últimos. Essa prática educativa produz um clima familiar hostil, estressado e sem diálogo, já que os filhos tentam proteger sua privacidade evitando falar com os pais sobre suas particularidades.

$\mathrm{Na}$ disciplina relaxada verifica-se o não cumprimento de regras estabelecidas pelos pais. Eles ameaçam os filhos e, quando se confrontam com comportamentos opositores e agressivos, omitem-se, não fazendo valer as regras que eles próprios determinaram (Gomide, 2003).

Considera-se abuso físico quando os pais machucam ou causam dor a seus filhos com a justificativa de que os estão educando. Para Gomide (2003), a prática do abuso físico pode gerar crianças apáticas, medrosas, desinteressadas.

\section{Inventá rio de Estilos Parentais}

A construção do Inventário se deu pelo entendimento de que a família é um núcleo importante de socialização e educação de crianças e que, dependendo dos comportamentos adotados pelos pais no processo educativo dos filhos, podemos encontrar crianças e adolescentes anti-sociais.

O Inventário é composto de 42 questões, sendo que cada uma consta de uma frase à qual a criança/adolescente deve responder indicando a freqüência com que a figura materna/paterna age (ia) conforme a situação descrita na frase. Assim, responde-se:

- NUNCA: se em 10 ocasiões, ele(a) agiu daquela forma de 0 a 2 vezes.

- ÀS VEZES: se em 10 ocasiões, ele(a) agiu daquela forma de 3 a 7 vezes.

- SEMPRE: se em 10 ocasiões, ele(a) agiu daquela forma de 8 a 10 vezes. 
As questões estão distribuídas de maneira que abranjam as sete práticas educativas: (A) monitoria positiva, (B) comportamento moral, (C) negligência, (D) punição inconsistente, (E) disciplina relaxada, (F) monitoria negativa e (G) abuso físico, sendo que a cada variável correspondem seis perguntas. A tabulação dos dados obtidos por meio do Inventário é feita utilizando-se a folha de resposta que contém as sete práticas educativas deste instrumento. Cada resposta NUNCA recebe pontuação 0 (zero); ÀS VEZES, pontuação 1 (um); e SEMPRE, pontuação 2 (dois). O cálculo do índice de estilo parental é feito pela subtração da soma das disciplinas negativas $(\mathrm{C}+\mathrm{D}+\mathrm{E}+\mathrm{F}+\mathrm{G})$ e da soma das positivas $(A+B)$, ou seja, IEP $=(A+B)-$ $(\mathrm{C}+\mathrm{D}+\mathrm{E}+\mathrm{F}+\mathrm{G})$.

\section{Estudos-piloto}

Segundo Cozby (2003), estudos-piloto são aqueles realizados geralmente com um pequeno número de participantes, com o objetivo de obterse uma primeira experiência acerca do instrumento, do método ou da compreensão da pesquisa por parte dos participantes. Eles permitem que o pesquisador realize alterações necessárias para otimização dos procedimentos da pesquisa.

O Inventário de Estilo Parental começou a ser elaborado em 1998, quando as questões referentes às práticas educativas estavam sendo objetos de avaliação. Buscou-se avaliar tanto o pertencimento das questões à categoria (práticas educativas) como a adequação da linguagem utilizada. Nesse primeiro momento, objetivou-se testar a eficácia de três medidas utilizadas. Aplicaram-se questionários a fim de levantar as práticas educativas adotadas pelos pais na educação dos filhos ( $\mathrm{n}=15$ casais); um jogo de palavras cruzadas ( $\mathrm{n}=15$ tríades pai-mãe-criança) para observar-se a interação entre eles de acordo com comportamentos categorizados; e foi realizada observação de comportamentos anti e pró-sociais das crianças em sala de aula. Os resultados iniciais indicaram a necessidade de reformulação do questionário aplicado a fim de tornar igual o número de alternativas referentes à monitoria positiva (31) e monitoria negativa (72). A correlação positiva entre monitoria negativa e observação de comportamento anti-social em sala de aula $(r=0,542, p=0,045)$ indicou que provavelmente as questões do questionário relativas a estilos parentais inadequados refletiram condutas anti-sociais nas escolas.

Feitas as necessárias modificações, o IEP foi testado em sua última versão durante os anos de 2002 e 2003. Esse estudo-piloto foi realizado em uma amostra com diferentes segmentos de adolescentes, procurando-se comparar diferenças entre gêneros (masculino e feminino), entre grupos de risco (adolescentes infratores e vítimas de abuso sexual) e de não-risco (estudantes) e entre classes sociais (estudantes de escolas públicas e particulares). O IEP foi aplicado em duas modalidades: quanto às práticas educativas paternas e quanto às práticas educativas maternas.

Gomide e Guimarães (2003) trabalharam com uma amostra de 163 adolescentes do sexo masculino pertencentes a dois grupos: um em situação de risco $(n=33)$ e outro de não-risco $(n=130)$.

Inicialmente, procurou-se testar a hipótese nula de que não havia diferenças de classe social quanto ao estilo parental, comparando o IEP dos alunos de escola pública com o de escola particular. O teste $t$ identificou diferença estatisticamente significativa entre as duas amostras em apenas uma situação: os estudantes da escola particular perceberam menor índice de monitoria negativa paterna do que o grupo da escola pública $(\mathrm{t}=2,294, \mathrm{p} \leq 0,05)$.

Num segundo momento, testou-se a hipótese nula de que não havia diferenças entre o grupo de risco com o grupo de não-risco quanto às práticas educativas adotadas. $\mathrm{O}$ teste $\mathrm{t}$ de Student indicou $t=-6,132 ; p \leq 0,001$ para os pais e de $t=-6,331 ; p \leq 0,001$ para as mães. Estas diferenças revelaram que o IEP identificou com sucesso estilos parentais de risco e de não-risco.

Para avaliar se havia consistência interna entre as questões do Inventário, aplicou-se o teste estatístico Alpha de Cronbach. Os valores alpha obtidos revelaram consistência interna em todas as práticas educativas, sendo que variaram de 0,46 para monitoria negativa materna a 0,8663 para comportamento moral paterno. Segundo Malhotra (2001), cargas superiores a 0,40 são suficientes para que o item faça parte do fator.

Gomide e Sabbag (2003) trabalharam com uma amostra de 162 adolescentes do sexo feminino pertencentes aos dois grupos: jovens em situação de risco $(n=22)$ e não-risco $(n=140)$.

Inicialmente, procurou-se testar a hipótese nula da semelhança nas práticas educativas pa- 
rentais entre as duas classes sociais: estudantes da escola pública e da escola particular. O teste $\mathrm{t}$ identificou diferenças estatisticamente significativas entre as duas amostras em cinco situações. As participantes das escolas particulares perceberam suas mães e seus pais com maiores índices de punição inconsistente do que as jovens das escolas públicas; também perceberam seus pais com maior índice de monitoria positiva e comportamento moral do que as segundas. As meninas das escolas públicas perceberam suas mães com maiores índices de monitoria negativa.

Testou-se a hipótese nula de que havia diferenças entre o grupo de risco com o grupo de não-risco quanto às práticas educativas. Esta análise mostrou que diferenças estatisticamente significativas foram encontradas para a maioria das práticas educativas e para o índice final, como esperado. No entanto, diferenças estatisticamente significativas foram obtidas em relação à monitoria positiva materna $(\mathrm{t}=-3,218, \mathrm{p}=0,002)$ e paterna $(\mathrm{t}=-3,959, \mathrm{p}=0,000)$, e ao comportamento moral paterno, em que o grupo de risco apresentou média bem inferior ao grupo de não-risco $(t=-2,881$, $\mathrm{p}=0,005)$. Nesse momento, o comportamento moral materno não apresentou diferença estatisticamente significativa possivelmente pelo baixo número de participantes da amostra de risco. Também foram encontradas diferenças estatisticamente significativas em práticas educativas negativas abuso físico paterno $(t=3,0807, p=0,000)$ e materno $(t=3,313, p=0,001)$ e negligência materna $(t=$ $4,336, p=0,000$ ), em que se observou uma média sensivelmente maior no grupo de risco em relação ao de não-risco. Além disso, o IEP, que representa o conjunto destas práticas parentais, obteve um valor igual a $\mathrm{t}=-3,125 ; \mathrm{p} \leq 0,005$ para as mães e de $\mathrm{t}=-3,029 ; \mathrm{p} \leq 0,005$ para os pais, ambos estatistica- mente significativos, demonstrando haver diferenças no estilo parental do grupo de risco para o de não-risco.

Nessa pesquisa também foi feita a análise da consistência interna das questões por meio do Alpha de Cronbach. Os valores alpha obtidos variaram de 0,4305 , para monitoria positiva materna, a 0,8726 , para abuso físico paterno.

Para verificar se havia diferença de gênero entre as amostras de Gomide e Guimarães (2003), Gomide e Sabbag (2003), testou-se a hipótese nula de que as práticas educativas parentais tanto do grupo de risco como de não-risco eram semelhantes. De modo geral, os resultados foram semelhantes, o que indicou que se poderia trabalhar com uma amostra única independentemente do sexo.

\section{Fidedignidade}

Cozby (2003) define fidedignidade como a consistência ou estabilidade de uma medida do comportamento, fornecendo uma medida estável da variável estudada. Assim, entende-se por fidedignidade a confiança que se tem numa medida, por ser ela capaz de repetir-se diversas vezes para a mesma variável apresentando baixo erro de mensuração.

No caso do IEP, um dos estudos sobre fidedignidade foi realizado por Carvalho (2003; Carvalho \& Gomide, 2005). A autora realizou uma pesquisa para investigar se a percepção dos pais é similar à dos filhos sobre as práticas educativas adotadas pela família, tendo como amostra 41 adolescentes autores de atos infracionais e seus respectivos pais. $O$ valor do IEP médio de cada membro da família foi negativo, como pode ser visto na tabela 1 .

\section{Tabela 1: Médias e desvios-padrão dos IEPs dos pais e filhos.}

\begin{tabular}{lll}
\hline Respostas ao IEP & Média & Desvio-padrão \\
\hline IEP materno (filho responde) & $-4,05$ & 8,87 \\
IEP paterno (filho responde) & $-3,59$ & 10,02 \\
IEP materno (mãe responde) & $-1,66$ & 9,01 \\
IEP paterno (pai responde) & $-3,8$ & 7,24 \\
\hline
\end{tabular}

Fonte: Carvalho (2003). 
Para verificar se os filhos percebiam as práticas educativas dos pais da mesma forma que eles as percebiam, foi aplicado o teste de Wilcoxon. Esse teste identificou que não houve diferença estatisticamente significativa em relação ao estilo parental, tanto para o IEP do filho em relação à mãe $(Z=1,44 ; \mathrm{p}=0,15)$ como para com o pai $(Z=$ $0,65 ; \mathrm{p}=0,51)$, isto é, a percepção das práticas parentais entre os membros da família mostrou-se similar, de maneira geral.

Nessa pesquisa, o teste de correlação de Spearman foi utilizado para analisar se as respostas dadas pelos filhos correlacionavam-se com as obtidas pelos IEPs dos pais. Testou-se a hipótese nula de que não existia correlação entre os resultados dos vários grupos de participantes. Como resultado obteve-se que, ao avaliar as práticas educativas materna e paterna, os filhos fizeram-no de maneira similar, ou seja, se negativa, a avaliação do estilo parental pelos filhos era consistente com a também avaliação negativa feita pelos próprios pais, e vice-versa.

A convergência de opinião entre as pessoas envolvidas, apoiada pela hipótese de que "os filhos percebem as práticas educativas parentais do mesmo modo que os pais as percebem", é muito importante nesta área de estudo, pois permite que pesquisadores confiem na fidedignidade dos dados coletados por intermédio dos filhos, pois estes provavelmente estarão refletindo também a opinião dos pais.

\section{Validade Interna e de Construto}

Pinheiro (2003) realizou uma pesquisa para observar se a maneira como o(a) filho(a) respondia ao questionário condizia com os comportamentos reais dos pais, utilizando para tal métodos qualitativos de entrevista (analisadas mediante os discursos-síntese) e de observação. A autora trabalhou com uma amostra de quatro famílias (12 participantes), sendo duas consideradas de nãorisco (IEP entre $+07 \mathrm{e}+15$ ) e duas de risco (IEP entre -08 e -34). Num primeiro momento, a autora realizou entrevistas de profundidade sobre "concepção de maternidade e paternidade" das mães e dos pais, respectivamente; "concepção de família"; e abordou as sete práticas educativas do IEP.

As entrevistas de profundidade com as famílias positivas e negativas demonstraram que o
IEP é um instrumento capaz de refletir a dinâmica familiar vigente. Por um lado, as famílias de nãorisco não fizeram menções de utilização de práticas como abuso físico e negligência, e poucas referências à monitoria negativa, punição inconsistente e disciplina relaxada, apresentando, por outro lado, inúmeros exemplos de monitoria positiva e comportamento moral. Já as famílias de risco se mostraram significativamente disfuncionais, apresentando altos índices em negligência e abuso físico, além de baixa monitoria positiva e comportamento moral. Tudo isso reflete a validade de construto do IEP, visto serem adequadas as definições operacionais para explicar o significado teórico das variáveis (Cozby, 2003).

\section{Validade de Construto: Comportamento Moral}

O comportamento moral é defendido pela literatura como uma das principais práticas inibidoras do desenvolvimento de comportamento antisocial (Loos, Ferreira \& Vasconcelos, 1999; Araújo, 1999; Comte-Sponville, 2000). No entanto, o conceito de comportamento moral é muito amplo e, em estudos preliminares do IEP, encontrou-se que mesmo os adolescentes de risco atribuíam altos indices de comportamento moral a seus pais (Gomide, 2003).

Em vista disso, para verificar quais variáveis seriam relevantes ao estudar a prática educativa Comportamento Moral e também analisar as diferenças e semelhanças em famílias de risco e de não-risco, Weber (2004) realizou uma pesquisa com 60 famílias, sendo 30 de risco e 30 de nãorisco.

Pretendeu-se observar se havia correlação entre IEPs positivos ou negativos e Índices de Comportamento Moral (ICM) elevados ou rebaixados. Para tal, foi elaborado um Questionário de Comportamento Moral e uma situação estruturada em que as variáveis estudadas seriam observadas e categorizadas qualitativamente por meio das respostas dos participantes às perguntas sobre a história "Cachinhos de Ouro" (Weber, 2004).

Com relação ao ICM, obteve-se que as famílias de não-risco apresentaram valores significativamente mais altos do que as de risco. O teste não-paramétrico de Mann-Whitney mostrou que as médias dos escores ao ICM para famílias de 
risco foram estatisticamente diferentes do que das famílias de não-risco (mãe $\mathrm{U}=291, \mathrm{p}=0,02$, e pai $\mathrm{U}=216,5, \mathrm{p}=0,001)$. Além disso, mediante aplicação do teste de correlação de Spearman, os escores do ICM dos adolescentes correlacionaram-se positivamente com os dos seus pais (mãe $r=0,3$, $\mathrm{p}=0,002$, e pai $\mathrm{r}=0,45, \mathrm{p} \leq 0,001)$.

A análise do discurso obtida por meio do debate da história "Cachinhos de Ouro" indicou diferenças qualitativas entre os grupos, sendo que o grupo de não-risco apresentou comportamentos socialmente mais flexíveis e adaptados.

\section{Validade Ex terna}

A validade externa de um estudo é o grau em que os resultados podem ser generalizados para outras populações ou situações (Cozby, 2003). Gomide, Pinheiro, Sabbag e Salvo (2005) correlacionaram o IEP com o Inventário de Habilidades Sociais - IHS, Inventário de Sintomas de Stress e Inventário Beck de Depressão, obtendo uma correlação positiva entre o IEP e o IHS - fator 2 ( $\mathrm{r}=$ $0,530, p=0,035)$, e negativa entre o IEP e o Inventário de Stress $(r=-0,523, \mathrm{p}=0,038)$ e de Depressão $(r=-0,707, p=0,002)$. Esses resultados indicam que em famílias de estilo parental positivo encontra-se um maior nível de Habilidades Sociais e menor índice de Estresse e Depressão, e vice-versa.

Com relação ao IHS (2001), dos oito membros das famílias com IEP negativo, apenas três apresentaram indicativo de repertório elaborado; já dentre as famílias de IEP positivo, sete membros apresentaram escores acima da média. Pela avaliação do Inventário de Depressão Beck (1997), não foram observados índices que acusassem depressão nas famílias com IEP positivo, enquanto, nas negativas, o valor chegou a $62,5 \%(n=5)$. Todos os membros das famílias de IEP negativo apresentaram índices relativos ao estresse, em oposição a apenas 25\% $(n=2)$ dos membros das famílias de IEP positivo, segundo o Inventário de Stress de Lipp (Gomide et al., 2005).

Por meio do teste não paramétrico de Mann-Whitney, obteve-se correlação positiva entre o IEP e o fator 2 (auto-afirmação na expressão de sentimentos positivos) do Inventário de Habilidades Sociais ( $\mathrm{U}=8$ e $\mathrm{p}=0,01$ ). O teste mostrou, ainda, uma correlação negativa entre o IEP e o Inventário de Stress de Lipp ( $U=7 ; p=0,005)$, e en- tre o IEP e o Inventário de Depressão de Beck $(\mathrm{U}=6,5$ e $\mathrm{p}=0,007)$. Analisando os escores obtidos, observou-se nos pais das famílias com IEP positivo ausência de depressão, ao passo que entre os membros das famílias com IEP negativo, 62,5\% $(\mathrm{n}=5)$ apresentaram escores indicativos dessa (Gomide et al., 2005).

Salvo, Silvares e Toni (2005) fizeram correlações do IEP com o CBCL (Child Behavior Check List - Achenbach, 1991). Os pesquisadores, mediante regressão múltipla (método stepwise), verificaram quais variáveis do IEP poderiam predizer cada uma das analisadas pelo CBCL. Constituíram a amostra 30 adolescentes alunos de uma ONG de Curitiba (PR) de ambos os sexos, com idade entre 11 e 13 anos. Além deles, um de seus pais respondeu aos questionários. De forma geral, os resultados indicaram coeficientes de determinação $\left(R^{2}\right)$ variando de 0,16 a $0,72\left(1 .^{\circ}\right.$ quartil: 0,20 ; mediana: 0,$31 ; 3 .^{\circ}$ quartil: 0,46$)$, o que significa que as variáveis preditoras (práticas educativas do IEP) explicaram de $16 \%$ a $72 \%$ da variância total das variáveis dependentes (CBCL). Em suma, os resultados sugerem que determinadas práticas educativas podem predizer, significativamente, o comportamento do adolescente.

\section{Perfil das Mães de Adolescentes de Risco}

Berri (2004) visou avaliar um programa de intervenção baseado nas práticas educativas do modelo de Gomide (2003) junto a mães de adolescentes em conflito com a lei. O programa foi desenvolvido com cinco mães, em oito sessões de uma hora e meia cada. Foram abordadas as práticas educativas do abuso físico e monitoria positi$\mathrm{va}$, fundamentalmente porque, a princípio, estas eram as principais dificuldades das mães, ou seja, elas utilizavam excessivamente punição física e não sabiam demonstrar afeto aos filhos.

$\mathrm{Na}$ análise das práticas educativas avaliadas, destacou-se que todas as práticas negativas do IEP foram identificadas nos discursos das mães. Vários relatos descreveram práticas referentes à monitoria negativa, sendo que o ambiente hostil, por vezes gerado, levava ao abuso físico. Este último mostrou-se intrinsecamente ligado à punição inconsistente, visto que o agente punidor, segundo essa prática, bate em função do humor e não das contingências vigentes. As práticas de abuso 
sexual e psicológico também se fizeram presentes nos discursos das mães; numa família um pai molestava as filhas e humilhava todos os outros membros.

Não foram encontradas expressões adequadas das práticas positivas. O comportamento moral não esteve presente nos relatos; ao contrário, uma família apresentava valores totalmente opostos a esses, o pai era alcoólatra, usuário de drogas, abusador sexual, psicológico e físico. Das cinco mães participantes, apenas duas demonstraram utilizar a monitoria positiva. No final do programa, foram comparadas as cartas escritas pelas mães aos seus filhos no início e no término do programa, podendo-se perceber que duas mães haviam diminuído os conteúdos agressivos de uma carta para outra; outras duas haviam aumentado um pouco tais conteúdos; e a outra não pôde ser comparada por não ter escrito a primeira carta. Sobre as duas mães que aumentaram seus conteúdos agressivos, Berri (2004) hipotetiza que uma mãe passou a ser mais assertiva com a intervenção, apresentando comportamentos agressivos primeiramente; e que a outra passou de uma posição fantasiosa sobre o filho a uma visão mais realista, demonstrando seus sentimentos de decepção em relação a ele. Nesse sentido, o programa também apresentou resultados importantes.

\section{Práticas Educativas e Consumo de Drogas}

Assis (2004) investigou a correlação entre abuso/dependência de substâncias e a presença das seguintes práticas educativas parentais: monitoria positiva, comportamento moral, negligência e abuso físico, as quais, segundo a literatura, indicam forte relação com o uso de drogas. Para tal, foram realizadas entrevistas com nove indivíduos (sendo oito do sexo masculino e um do sexo feminino) dependentes e (ou) abusadores de substâncias, com idade entre 11 a 21 anos. Os instrumentos utilizados foram o IEP e uma entrevista semi-estruturada abordando as características das práticas educativas citadas dos pais dos adolescentes abusadores de substâncias.

Os IEPs das díades estudadas variaram de -26 a +7 ; no entanto, em todas elas ao menos um dos membros do casal apresentou IEP negati- vo. A única exceção foi uma díade que apresentou IEP 0 (zero) para o pai e +3 para a mãe.

Quanto à negligência, verificou-se que todos os pais e 66\% das mães apresentaram índices de risco nessa prática. Os discursos registrados pela pesquisadora na entrevista semi-estruturada indicaram que, provavelmente, a maioria dos adolescentes da amostra não recebeu de ambos os pais o carinho, a atenção e a satisfação de suas necessidades psicológicas (Assis, 2004). Em relação à prática abuso físico, verificou-se que todas as mães e $75 \%$ dos pais apresentaram taxas de risco de abuso físico.

No que diz respeito ao comportamento moral, obteve-se que $44 \%$ das mães apresentaram escores de risco e $37 \%$ dos pais índices de comportamento moral abaixo da média, entre os percentis de 30 a 50. Porém, o que se observou mediante a análise dos discursos e dos valores da prática de monitoria negativa é que as mães e pais que obtiveram bons escores nessa prática também marcaram alta pontuação em monitoria negativa.

Setenta e cinco por cento (75\%) dos pais e $55,5 \%$ das mães apresentaram índices de risco de monitoria positiva. Isso quer dizer que a demonstração de afeto, carinho e a preocupação com o filho são deficitárias.

\section{Validação do IEP}

Com o objetivo de validar o IEP, Gomide e Sampaio (2004) e Gomide e Maggi (2004) aplicaram o referido instrumento em amostras de risco e não-risco para serem comparadas, e para que os dados coletados possibilitassem a construção de tabelas normativas. Assim, Gomide e Sampaio (2004) utilizaram uma amostra de não-risco composta por 633 alunos oriundos de dois colégios de Curitiba: 452 de um colégio particular e 181 de um colégio estadual, sendo ao todo 309 do sexo masculino e 324 do sexo feminino. Suas idades variavam de 13 a 16 anos. Gomide e Maggi (2004) desenvolveram o mesmo estudo com base na aplicação do IEP numa população de risco. A amostra constitui-se de 136 sujeitos, sendo 107 do sexo masculino e 29 do feminino, oriundos de uma instituição para jovens infratores e abrigos para adolescentes vítimas de abuso. As médias de cada prática de ambos os grupos podem ser observadas na tabela 2, a seguir. 
Tabela 2. Médias das práticas e índices paternos e maternos da população de risco e não-risco.

\begin{tabular}{l|l|l|l|l|l|l|l|l}
\hline $\begin{array}{l}\text { Figura } \\
\text { parental }\end{array}$ & $\begin{array}{l}\text { Monitoria } \\
\text { positiva }\end{array}$ & $\begin{array}{l}\text { Comporta- } \\
\text { mento moral }\end{array}$ & Negligência & $\begin{array}{l}\text { Punição } \\
\text { inconsis- } \\
\text { tente }\end{array}$ & $\begin{array}{l}\text { Disciplina } \\
\text { relaxada }\end{array}$ & $\begin{array}{l}\text { Monitoria } \\
\text { negativa }\end{array}$ & $\begin{array}{l}\text { Abuso } \\
\text { físico }\end{array}$ & $\begin{array}{l}\text { Índices } \\
\text { gerais }\end{array}$ \\
\hline Mãe não-risco & 9,83 & 8,87 & 2,37 & 3,58 & 3,19 & 5,54 & 1,05 & 2,97 \\
Mãe risco & 7,28 & 7,61 & 5,16 & 5,17 & 5,25 & 6,57 & 4,44 & $-11,70$ \\
Pai não-risco & 8,61 & 8,33 & 3,07 & 3,18 & 2,76 & 4,54 & 1,22 & 2,18 \\
Pai risco & 7,02 & 7,57 & 6,12 & 5,92 & 5,70 & 6,69 & 5,58 & $-15,04$ \\
\hline
\end{tabular}

Fonte: Gomide \& Sampaio (2004).

Todas as médias das práticas positivas da população de risco foram menores do que as de nãorisco, tanto para pais quanto para mães, apontando diferenças estatisticamente significativas em todas elas.

Os dados obtidos foram submetidos ao teste $\mathrm{t}$ de Student para amostras independentes para avaliar a existência de diferenças entre o grupo de não-risco de Gomide e Sampaio (2004) e o de risco de Gomide e Maggi (2004). Com exceção do comportamento moral $(\mathrm{p}=0,014)$ do pai, as demais práticas educativas foram estatisticamente diferentes nos dois grupos amostrais ao nível de $0,01 \%$ ( $p<0,0001)$, o que representa uma forte indicação de que o IEP consegue detectar as práticas educativas de risco em adolescentes considerados de risco. Mesmo o comportamento moral, como prática educativa do pai, não deixou de ser estatisticamente diferente nos dois grupos amostrais, porém ao nível de significância de 2,5\% ( $\mathrm{p}<0,025)$.

Tendo-se em mão tais dados, pôde-se elaborar duas tabelas normativas que permitem identificar se o respondente está ou não num nível de risco e, se o estiver, qual a gravidade dele. As duas tabelas (tabelas 3 e 4), uma referente às práticas maternas e outra às paternas, devem ser consultadas separadamente. Assim, após a obtenção dos valores de cada prática e do índice geral, encontra-se na tabela o percentil equivalente, que deverá ser interpretado de acordo com os números obtidos.

\section{Tabela 3. Dados normativos das práticas educativas maternas.}

\begin{tabular}{c|c|c|c|c|c|c|c|c}
\hline Percentis & IEP & $\begin{array}{c}\text { monitoria } \\
\text { positiva }\end{array}$ & $\begin{array}{c}\text { comportamento } \\
\text { moral }\end{array}$ & $\begin{array}{c}\text { abuso } \\
\text { físico }\end{array}$ & $\begin{array}{c}\text { negli- } \\
\text { gência }\end{array}$ & $\begin{array}{c}\text { monitoria } \\
\text { negativa }\end{array}$ & $\begin{array}{c}\text { punição } \\
\text { inconsistente }\end{array}$ & $\begin{array}{c}\text { disciplina } \\
\text { relaxada }\end{array}$ \\
\hline 99 & 19 & 12 & 12 & 0 & 0 & 1 & 0 & 0 \\
95 & 15 & 12 & 12 & 0 & 0 & 2 & 0 & 0 \\
90 & 13 & 12 & 12 & 0 & 0 & 3 & 1 & 0 \\
85 & 12 & 12 & 11 & 0 & 0 & 3 & 1 & 1 \\
80 & 11 & 12 & 11 & 0 & 1 & 4 & 2 & 1 \\
75 & 9 & 11 & 11 & 0 & 1 & 4 & 2 & 2 \\
70 & 8 & 11 & 11 & 0 & 1 & 4 & 2 & 2 \\
65 & 7 & 11 & 10 & 0 & 1 & 5 & 2 & 2 \\
60 & 6 & 11 & 10 & 0 & 1 & 5 & 3 & 2 \\
55 & 5 & 10 & 10 & 0 & 2 & 5 & 3 & 3 \\
50 & 4 & 10 & 9 & 0 & 2 & 5 & 3 & 3 \\
45 & 3 & 10 & 9 & 1 & 2 & 6 & 4 & 3 \\
40 & 2 & 10 & 9 & 1 & 2 & 6 & 4 & 3 \\
35 & 1 & 9 & 8 & 1 & 3 & 6 & 4 & 4 \\
30 & 0 & 9 & 8 & 1 & 3 & 7 & 5 & 4 \\
25 & -2 & 8 & 7 & 1 & 3 & 7 & 5 & 4 \\
20 & -3 & 8 & 7 & 2 & 4 & 7 & 5 & 5 \\
15 & -6 & 7 & 6 & 2 & 4 & 8 & 6 & 5 \\
10 & -9 & 7 & 5 & 3 & 5 & 8 & 7 & 6 \\
5 & -14 & 6 & 4 & 5 & 7 & 9 & 8 & 7 \\
1 & -22 & 3 & 2 & 8 & 10 & 11 & 9 & 10 \\
\hline Média & $\mathbf{2 , 9 7}$ & $\mathbf{9 , 8 3}$ & $\mathbf{8 , 8 7}$ & $\mathbf{1 , 0 5}$ & $\mathbf{2 , 3 7}$ & $\mathbf{5 , 5 4}$ & $\mathbf{3 , 5 8}$ & $\mathbf{3 , 1 9}$ \\
\hline Desvio & $\mathbf{8 , 7 9}$ & $\mathbf{2 , 4 1}$ & $\mathbf{2 , 4 9}$ & $\mathbf{1 , 6 8}$ & $\mathbf{2 , 1 7}$ & $\mathbf{2 , 2 7}$ & $\mathbf{2 , 2 6}$ & $\mathbf{2 , 2 1}$ \\
padrão & & & & & & & \\
\hline
\end{tabular}

Fonte: Gomide (2006). 
A seguir, os dados normativos referentes às práticas paternas.

\section{Tabela 4. Dados normativos das práticas paternas.}

\begin{tabular}{c|c|c|c|c|c|c|c|c}
\hline Percentis & IEP & $\begin{array}{c}\text { monitoria } \\
\text { positiva }\end{array}$ & $\begin{array}{c}\text { comportamento } \\
\text { moral }\end{array}$ & $\begin{array}{c}\text { abuso } \\
\text { físico }\end{array}$ & $\begin{array}{c}\text { negli- } \\
\text { gência }\end{array}$ & $\begin{array}{c}\text { monitoria } \\
\text { negativa }\end{array}$ & $\begin{array}{c}\text { punição } \\
\text { inconsistente }\end{array}$ & $\begin{array}{c}\text { disciplina } \\
\text { relaxada }\end{array}$ \\
\hline 99 & 20 & 12 & 12 & 0 & 0 & 0 & 0 & 0 \\
95 & 15 & 12 & 12 & 0 & 0 & 1 & 0 & 0 \\
90 & 13 & 12 & 12 & 0 & 0 & 2 & 0 & 0 \\
85 & 12 & 12 & 11 & 0 & 1 & 2 & 1 & 0 \\
80 & 10 & 11 & 11 & 0 & 1 & 2 & 1 & 1 \\
75 & 9 & 11 & 11 & 0 & 1 & 3 & 1 & 1 \\
70 & 8 & 11 & 10 & 0 & 1 & 3 & 2 & 1 \\
65 & 7 & 10 & 10 & 0 & 2 & 4 & 2 & 2 \\
60 & 6 & 10 & 10 & 0 & 2 & 4 & 2 & 2 \\
55 & 5 & 10 & 9 & 0 & 2 & 4 & 3 & 2 \\
50 & 4 & 9 & 9 & 0 & 2 & 4 & 3 & 3 \\
45 & 3 & 9 & 8 & 1 & 3 & 5 & 3 & 3 \\
40 & 2 & 8 & 8 & 1 & 3 & 5 & 4 & 3 \\
35 & 0 & 8 & 8 & 1 & 3 & 5 & 4 & 3 \\
30 & -2 & 7 & 7 & 1 & 4 & 6 & 4 & 4 \\
25 & -3 & 7 & 6 & 2 & 4 & 6 & 5 & 4 \\
20 & -6 & 6 & 6 & 2 & 5 & 7 & 5 & 4 \\
15 & -8 & 5 & 5 & 3 & 6 & 7 & 6 & 5 \\
10 & -11 & 4 & 4 & 4 & 7 & 8 & 6 & 6 \\
5 & -16 & 3 & 3 & 6 & 8 & 9 & 7 & 7 \\
1 & -25 & 2 & 0 & 8 & 11 & 10 & 10 & 9 \\
\hline Média & $\mathbf{2 , 1 8}$ & $\mathbf{8 , 6 1}$ & $\mathbf{8 , 3 3}$ & $\mathbf{1 , 2 2}$ & $\mathbf{3 , 0 7}$ & $\mathbf{4 , 5 4}$ & $\mathbf{3 , 1 8}$ & $\mathbf{2 , 7 6}$ \\
\hline $\mathbf{D e s v i o}$ \\
padrão & $\mathbf{9 , 5 6}$ & $\mathbf{2 , 9 3}$ & $\mathbf{2 , 9 9}$ & $\mathbf{1 , 9 4}$ & $\mathbf{2 , 5 6}$ & $\mathbf{2 , 3 6}$ & $\mathbf{2 , 3 5}$ & $\mathbf{2 , 2 3}$ \\
\hline
\end{tabular}

Fonte: Gomide (2006).

A interpretação dos resultados do IEP segue o valor do percentil obtido. Assim, pode-se considerar que os percentis:

De 75 a 99 - referem-se a um estilo parental ótimo, com presença marcante das práticas parentais positivas e ausência das práticas negativas.

De 55 a 70 - referem-se a um estilo parental bom, acima da média, porém aconselhase a leitura de livros de orientação para pais visando ao aprimoramento das práticas parentais.

De 30 a 50 - referem-se a um estilo parental bom, porém abaixo da média. Aconselha-se a participação em grupos de treinamento para pais.

Abaixo de 25 - referem-se a um estilo parental de risco. Aconselha-se a participação em Programas de Intervenção especialmente desenvolvidos para pais com dificuldades em Práticas Educativas ou Terapia de Grupo, de casal ou indi- vidual em que possam ser enfocadas as conseqüências do uso de práticas negativas em detrimento das positivas.

As tabelas normativas construídas identificam o nível de risco apresentado pelo respondente de modo a considerar as práticas parentais utilizadas por meio dos índices obtidos para cada uma delas, sendo posteriormente calculado o índice geral.

\section{Conclusão}

Passados oito anos de pesquisas na área, foi realizada a validação do Inventário de Estilos Parentais - IEP - dentro dos parâmetros psicométricos necessários. O Conselho Federal de Psicologia, em julho de 2005, deu parecer favorável à utilização do IEP como teste psicológico. Em mar- 
ço de 2006, a Editora Vozes publicou o Inventário de Estilos Parentais - Modelo Teórico, Manual de Aplicação, Apuração e Interpretação. Esse instrumento vem auxiliar os profissionais que trabalham com populações de risco a identificar os déficits educacionais presentes nas famílias, favorecendo o encaminhamento para programas de orientação, treinamento ou de intervenção clínica. O Inventário pode ser utilizado como medida de pré e póstestes, coleta de dados, para correlações com outros instrumentos, avaliação de nível de risco etc. O IEP também apresenta a vantagem de rápida aplicação e uma linguagem de fácil acesso, além de uma forma descomplicada de analisar dados. Ressalta-se que ele é o primeiro instrumento brasileiro validado que possibilita a avaliação de sujeitos de risco, sendo, portanto, de grande demanda por parte de pesquisadores e profissionais da área.

\section{Referências}

Araújo, U. F. (1999). Conto de escola: A vergonha como um regulador moral. São Paulo: Moderna.

Assis, C. L. (2004). Drogadição e práticas parentais associadas. Dissertação de Mestrado. Programa de pós-graduação em psicologia da infância e da adolescência, Universidade Federal do Paraná, Curitiba.

Berri, G. (2004). Programa de intervenção em práticas parentais para mães de adolescentes em conflito com a lei. Dissertação de Mestrado. Programa de Pós-graduação em psicologia da infância e da adolescência, Universidade Federal do Paraná, Curitiba.

Carvalho, M. C. N. (2003). Efeitos das práticas educativas parentais sobre o comportamento infrator de adolescentes. Dissertação de Mestrado. Programa de pós-graduação em psicologia da infância e da adolescência, Universidade Federal do Paraná, Curitiba.

Carvalho, M. C. N., \& Gomide, P. I. C. (2005). Práticas educativas parentais em famílias de adolescentes em conflito com a lei. Estudos em psicologia, 22(3), 263-276.

Comte-Sponville, A. (2000). Pequeno tratado das grandes virtudes. São Paulo: Martins Fontes.
Cozby, P. (2003). Métodos de pesquisa em ciências do comportamento. São Paulo: Atlas.

Dishion, T. J., \& Mcmahon, R. J. (1998). Parental monitoring and the prevention of child and adolescent problem behavior. A conceptual and empirical formulation. Clinical child and family psychology review, 1(1), 61-75.

Dodge, K. A., Pettit, G. S., \& Battes, J. E. (1994). Socialization mediators of the relation between socioeconomic status and child conduct problems. Child Development, 65, 649-665.

Gomide, P. I. C. (2001). Efeito das práticas educativas no desenvolvimento do comportamento anti-social. In: M. L. Marinho \& V. E. Caballo (orgs). Psicologia clínica e da saúde. Londrina (Brasil), Granada (Espanha): UEL.

Gomide, P. I. C. (2003). Estilos parentais e comportamento anti-social. In: A. Del Prette e Z. Del Prette (orgs). Habilidades sociais, desenvolvimento e aprendizagem: Questões conceituais, avaliação e intervenção. Campinas: Alínea.

Gomide, P. I. C., \& Guimarães, A. M. (2003). [Efeitos das práticas educativas sobre o comportamento anti-social em crianças e adolescentes - Validação do Inventário de Estilos Parentais (IEP): aplicação em sujeitos do sexo masculino]. (Relatório de pesquisa PIBIC/CNPq). Curitiba: UFPR. Unpublished.

Gomide, P. I. C., \& Sabbag, G. M. (2003) [Efeitos das práticas educativas sobre o comportamento anti-social em crianças e adolescentes - Validação do Inventário de Estilos Parentais (IEP): aplicação em sujeitos do sexo feminino]. (Relatório de pesquisa PIBIC/CNPq). Curitiba: UFPR. Unpublished.

Gomide, P. I. C., \& Maggi, C. (2004). [Estilos parentais e comportamento anti-social Validação do Inventário de Estilos Parentais (IEP) - aplicação em população de risco]. (Relatório de pesquisa PIBIC/CNPq). Curitiba: UFPR. Unpublished.

Gomide, P. I. C., \& Sampaio, I. T. A. (2004) [Estilos parentais e comportamento anti-social - Validação do Inventário de Estilos Parentais (IEP) - aplicação em população de nãorisco]. (Relatório de pesquisa PIBIC/CNPq). Curitiba: UFPR. Unpublished. 
Gomide, P. I. C., Pinheiro, D. P. N., Sabbag, G. M., \& Salvo, C. G. (2005). Correlação entre práticas educativas, estresse, depressão e habilidades sociais. Psico-USF, 10(2), 169-178.

Gomide, P. I. C. (2006). Inventário de estilos parentais: Modelo teórico, manual de aplicação, apuração e interpretação. Petrópolis: Vozes.

Hoffman, M. L. (1975). Moral internalization, parental power and the nature of parent-child interaction. Developmental Psychology, 11(2), 228-239.

Loos, H. \& Ferreira, S. P. A., \& Vasconcelos, F. C. (1999). Julgamento moral: estudo comparativo entre crianças institucionalizadas e crianças de comunidade de baixa renda com relação à emergência do sentimento de culpa. Psicologia: Reflexão e crítica, 12(1), 47-70.

Malhotra, N. K. (2001). Pesquisa de marketing: Uma orientação aplicada. Porto Alegre: Bookman.

Mussen, P. H., Conger, J. J., \& Kagan, J. (1974). Desenvolvimento e personalidade da criança. São Paulo: Harbra.

Pasquali, L. (2003). Psicometria teoria dos testes na psicologia e na educação. Petrópolis: Vozes.

Patterson, G. R., Reid, J. B., \& Dishion, T. J. (1992). Antisocial boys. USA: Castalia.

Pettit, G., Laird, R. D., Dodge, K. A., Bates, J., \& Criss, M. (2001). Antecedents and behavior-problem outcomes of parental monitoring and psychological control in early adolescence. Child Development, 72, 583-598.
Pinheiro, D. P. N. (2003). Estilo parental: Uma análise qualitativa. Dissertação de Mestrado. Programa de Pós-graduação em psicologia da infância e da adolescência, Universidade Federal do Paraná, Curitiba.

Reppold, C. T., Pacheco, J., Bardagi, M., \& Hutz, C. S. (2002). Prevenção de problemas de comportamento e desenvolvimento de competências psicossociais em crianças e adolescentes: uma análise das práticas educativas e dos estilos parentais. In: S. C. Hutz. Situações de risco e vulnerabilidade na infância e na adolescência: Aspectos teóricos e estratégia de intervenção. São Paulo: Casa do Psicólogo.

Salvo, C. G., Silvares, E. M., \& Toni, P. M. (2005). Práticas educativas como preditoras de problemas de comportamento e competência social. Psicologia em Estudo, 22(2), 187-196.

Stattin, H., \& Kerr, M. (2000). Parental Monitoring: A reinterpretation. Child Development, 71, 1072-1085.

Weber, L. (2004). Efeitos do comportamento moral dos pais sobre o comportamento moral dos filhos adolescentes. Dissertação de mestrado, programa de pós-graduação em psicologia da infância e adolescência, Universidade Federal do Paraná, Curitiba.

Recebido em/received in: 07/06/2006 Aprovado em/approved in: 11/09/2006 